Aus der Universităts-Hautklinik in Bonn.

(Direktor: Prof. E. Hoffmann.)

\title{
Zur Kenntnis der Alopecia diffusa nach Grippe.
}

Von Dr. E. Zurhelle.

Die lebensbedrohlichen Erscheinungen an den inneren Organen stehen bei Grippe- und Influenzaepidemien so sehr im Vordergrunde des Interesses, daß über die dermatologischen Nebenerscheinungen dieser Erkrankung wenig bekannt geworden ist.

Aus dem Jahre 1890 liegt eine Zusammenstellung von Schwimmer (W. m. W.) über die komplizierenden Hautaffektionen vor. Er unterscheidet Herpesformen, Erytheme, Urtikaria, sah einige Male ein morbillöses Exanthem und eine Psoriasiseruption als eine Folge der Influenza und zieht bei allen diesen Erkrankungen, seinen theoretischen Anschauungen entsprechend, eine angioneurotische Affektion in Erwägung. Leichtenstern in Nothnagels ,Spezieller Pathologie und Therapie" gibt einen historischen Rückblick über die bisher bei Influenza beschriebenen Exantheme und berichtet, daß sie - soweit sie nicht auf Arznei-, insbesondere Antipyrineinwirkung beruhen - als eine durch Grippotoxine bedingte Angioneurose, "Vasomotorenlähmung" angesehen werden. Er fand in $9 \%$ seiner Fälle ein feinfleckiges Exanthem und èrwähnt die interessanten, acht Tage nach der Erkrankung auftretenden, vermutlich durch Grippotoxine hervorgerufenen Spätexantheme. Ein von Rumpel (im II. Teil der Unna-Festschrift) beschriebenes großfleckiges Exanthem bei grippeartigen Erkrankungen möehte ich wegen der komplizierenden Darmerscheinungen in seinen Fällen nur erwăhnen. Von der letzten Grippeepidemie berichtet Hain iss (W. m. W. 1919 Nr. 8) über scharlachähnliche Exantheme. Sonst fand ich nur kürzere Angaben von Schwenkenbecher und Wachter (M. m. W. 1919 Nr. 2)

Um so mehr steht jetzt nach Ablauf der letzten Epidemie im Oktober und November 1918 der darauf zurückzuführende Haarausfall im Vordergrunde des Interesses.

Wenn es auch bekannt war, daß nach akuten Fieberkrankheiten, Influenza, Scharlach, Typhus, Fleckfieber, Blind-

1) Sie wird, ebenso wie das"Amidometer, von Rich. Kallmeyer \& Co.. Berlin, Johannisstr. 20/21, hergestellt: 
darmentzündung usw. plötzlich ein diffuser Haarausfall auftritt, so sind die näheren Vorgänge dabei doch wohl so wenig Allgemeingut der Dermatologen geworden, daß es sich verlohnt, kurz noch einmal darauf einzugehen, um so mehr, als seit Anfang Januar 1919 eine stets zunehmende Zahl von Patienten mit der Angabe erscheint, im Oktober resp. November 1918 eine schwere Grippeerkrankung durchgemacht und vor kurzem ganz plötzlich einen, mitunter ä̉ußerst starken, Haarausfall bemerkt zu haben.

Die Haare zeigen bei der mikroskopischen Untersuchung teils eine Verdünnung und Aufhellung am Wurzelende, teils fehlt sie, bei andern ist diese Veränderung ca. $40 \mathrm{~mm}$ vom Ende entfernt.

Die verschiedenen Bilder sind so verwirrend, daß es vielleicht berechtigt ist, die bisherigen Untersuchungen von Pohl, Matsuura und Pinkus ${ }^{1}$ ) mit meinen eigenen, aus etwa 30-40 Fällen ausgesuchten Beobachtungen kurz zusammenzustellen. ${ }^{2}$ )

Die Haare wurden (wenn möglich ausgefallene und ausgezogene gesondert) in Zedernholzöl eingelegt, zum Teil mit der Wurzel, zum Teil mit dem Schaft nach der von Pinkus angegebenen Methode unter einem besonders großen Deckglas, um Haarspitze und -wurzel zum Verschieben des Haares in der Längsrichtung benutzen zu können. Vereinzelte Haare wurden auch nach der Methode Matsuuras in kleine Stückchen geschnitten und untersucht. Das Ergebnis ist, daß jede stärkere Allgemeinerkrankinu in Form einer Ernährungsstörung oder Toxinwirkung auf das Kopfhaar (Woll- und Borstenhaare waren nach unserer Beobachtung hicht befallen) $\mathrm{zu}$ einer Verdünnung, Aufhellung und Markveränderung (Markschwund) desselben führt, die entweder vorübergehend ist oder mehr oder weniger schnell ein Absterben des Haares zur Folge hat.

Das ausgefallene Haar endet in solchen Fällen mit einem Kolben (Kolbenhaar), der entsprechend der Dauer der schädigenden Einwirkung bis zum völligen Absterben eine mehr oder weniger lange Depigmentierung am Wurzelende aufweist. Derartige Kolbenhaare fanden sich in überwiegender Mehrzahl in folgenden Fällen:

Beobachtung 1 (J.-Nr. 2734). 13 jähriges, dunkelblondes Mädchen litt Mitte November drei Wochen lang an Grippe ohne Lungenentzündung, aber mit sehr hohem Fieber (von Arzt und Krankenschweseter behandelt). Es erschien am 27. Januar wegen Haarausfalls seit acht Tagen, wobei bereits über die Hälfte der Haare ausgefallen sei. Es fanden sich deutliche Nagelveränderungen (Querfurche) am zweiten bis fünften Finger beiderseits.

Die Differenz zwischen Beginn der Erkrankung und Beginn des Haarausfalls betrug etwa 70 Tage. Mikroskopisch erwiesen sich die Haare durchweg als Kolbenhaare, die am Wurzelende $1,10-1,30 \mathrm{~mm}$ weit depigmentiert, verschmälert und marklos waren.

Beobachtung 2 (J.-Nr. 127). 27jährige Studentin mit schwarzen Haaren, darunter vereinzelten weißen, machte im Oktober und November Grippe mit Lungenentzündung (Höchsttemperatur 40,5) durch. Seit Mitte November Haarausfall, in letzter Zeit wesentlich verschlimmert. Patientin $\mathrm{kam}$ am $27 . \mathrm{Ja}-$ nuar in die Poliklinik mit sehr weit vorgeschrittener Haarverdünnung.

Beausche Linien waren an den Nägeln der zweiten bis fünften Finger beiderseits deutlich sichtbar; eine Eindellung bestand aber nicht.

Der zeitliche Abstand zwischen Erkrankung und Konsultation betrug etwa 90 Tage.

Mikroskopisch fanden sich Kolbenhaare, die durchweg am Wurzelende $0,8 \mathrm{~mm}$, andere auch bis $\mathrm{zu} 2,4 \mathrm{~mm}$ aufgehellt und von 0,085 auf 0,06 resp. von 0,06 auf $0,0425 \mathrm{~mm}$ verdünnt waren. Gleichzeitig war vőlliger Markschwund eingetreten.

Beobachtung 3 betrifft eine Wärterin unserer Klinik, die am 10. Oktober 1918 an sehr hochgradiger Grippe erkrankte, gleich zu Beginn mit hohem Fieber. Sie bemerkte seit Anfang Januar einen hochgradigen Haarausfall. Bei ihr fanden sich Anfang Februar deutliche Beausche Limen an beiden Daumenägeln. Die vorwiegend vorhandenen, meist marklosen Kolbenhaare waren zum größten Teile bis zum Wurzelende pigmentiert, nicht wesentlich verdünnt.

Im Gegensatz dazu fanden sich bei der Angestellten einer benachbarten Klinik (Beobachtung 4) gleichfalls vorwiegend Kolbenhaare, aber vor dem Ende dieser Haare fanden sich längere pigmentlose Strecken von durchschnittlich 1,2-3,2 $\mathrm{mm}$ Länge und einer Verdünnung von 0,065 auf 0,05 resp. 0,055 auf $0,050 \mathrm{~mm}$. Diese Patientin machte im November $19183^{1} !_{2}$ Wochen lang Grippe durch mit länger anhaltendem Fieber um 40, sodaß der Verdacht auf AbszeB entstand. Drei Wochen später trat ein zunehmender, hochgradiger Harrausfall ein.

In allen diesen Fällen war es zum Absterben des Haares gekommen. Wie beschrieben, kann man selbst noch an den ab-

1) F. Pinkus, Die Einwirkung von Krankheiten auf das Kopfhaar des Menschen. Verlag Karger 1917.

2) Nach.Fertigstellung erschienen zum gleichen Thema die Arbeiten von M e ri a n, Schweiz. Korr.Bl. 1919 Nr. 5, Pi n ǩus. M. Kl. 1919 Nr. 8, und S a lewskg, !M. m. W. 1919 Nr. 14. gestorbenen Haaren erkennen, ob die Erkrankung sofort (wie bei Beobachtung 3) oder nach kurzer (Beobachtung 1 und 2) oder längerer Stởrung (Beobachtung 4) zum völligen Wachstumsstillstand geführt hatte.

Gerade der letzte Fall, wo offenbar die Mehrzahi der Haare erst nach längerem Bestehen der Erkrankung abgestorben war, leitet uns za den Fällen über, wo zwar gleichfalls abgestorbene Kolbenhaare zu einer Verminderung des Haares führen, die überwiegende Mehrzahl der Haare aber als Papillenhaare die Frkrankung überstehen und bei der Untersuchung sukkulente Wurzelscheiden und ein wohl ausgebildetes und pigmentiertes, auch - soweit die. Haare überhaupt markhaltig sind - markhaltiges Wurzelende aufweisen, in größerer Entfernung von diesen aber eine mehr oder weniger ausgeprägte Ernährungsstörung zeigen.

So machte Frau M. K. (Beobachtung 5, J.-Nr. 2580), 23 Jahre alt, vom 6 . bis 16. resp. 17. Oktober Grippe mit sehr hohem Fieber durch, wobei ein Lungenflügel angegriffen war.

Zwei Monate später bemerkte sie einen Haarausfall. Bei der Untersuchung am 6. Februar 1919 fanden sich starke Beausche Linien an beiden Daumen, am ersten Zeigefinger und am ersten Mittelfinger. Mikroskopisch zeigte ein Haar in 4,4 cm Entfernung vom Wurzelende eine $2 \mathrm{~mm}$ lange Verdünnung und starke Aufhellung seiner starken Pigmentierung, ein anderes in $3,9 \mathrm{~cm}$ Entfernung eine 3,6 $\mathrm{mm}$ lange Verdünnung. Das Mark war an diesen Stellen völlig geschwunden, die Haardicke betrug 0,0425 statt 0,06 resp. 0,0375 statt $0,0425 \mathrm{~mm}$.

Die Differenzierung zwischen Ende der Erkrankung und Untersuchung betrug über 100 Tage, was bei der genannten Entfernung von 4,4 resp. $3,9 \mathrm{~cm}$ einem ungefähren Wachstum von $0,4 \mathrm{~mm}$ pro Tag entsprechen würde; die Länge der Verdünnung dividiert durch die Zahl der Krankheitstage ergäbe für die Zeit der Erkrankung ein Wachstum von täglich $0,2-0,36 \mathrm{~mm}$.

Um ähnliche Verhältnisse handelt es sich bei Frl. M. M. (Beobachtung 6, J.-Nr. 2793), 16 Jahre alt, hellblond. Sie litt Mitte Oktober acht Tage lang an Grippe, kam am 3. Februar 1919 wegen mäßigen, seit acht Tagen bestehenden Haarausfalls zur Untersuchung. Die Differenz betrug also abermals etwa 100 Tage. Es fanden sich Beausche Linien an den Daumen. Die Untersuchung ergab zahlreiche, meist marklose Kolbenhaare. Ein Haar zeigte in $28 \mathrm{~mm}$ Entfernung vom Wurzelende eine 3,2 $\mathrm{mm}$ lange, starke Aufhellung und Verdünnung von 0,0775 auf $0,0675 \mathrm{~mm}$, ein anderes in $28 \mathrm{~mm}$ Entfernung eine 1,6 $\mathrm{mm}$ lange Verdünnung und Aufhellung. Das ergab für das Wachstum während der Erkrankung $0,4-0,2 \mathrm{~mm}$ pro Tag, für dasjenige nach der Erkrankung eine erhebliche Verlangsamung, nämlich nur $0,28 \mathrm{~mm}$.

Eine weitere Patientin endlich, Frau M. St. (Beobachtung 7, J.-Nr. 2826), 28 Jahre alt, dunkelbraun, lag im Oktober und November acht Wochen $(=56$ Tage $)$ mit schwerer Lungenentzündung und Herzschwäche dạrnieder. Sie kam am 6. Februar zur Untersuchung, da seit drei Wochen plötzlich Haarausfall (von bisher $160 \mathrm{~g}$ Gesamtgewicht) eingesetzt hatte.

Es waren also seit dem Ende der Erkrankung etwa 75 Tage vergangen. An allen Fingern waren deưtliche Beausche Linien sichtbar. Ein Haar zeigte in $33 \mathrm{~mm}$ Entfernung eine $5,6 \mathrm{~mm}$ lange, stark depigmentierte, von 0,08 auf $0,0625 \mathrm{~mm}$ verdunnte Stelle, ein anderes in $29 \mathrm{~mm}$ Entfernung eine $1,9 \mathrm{~mm}$ lange, gleiche, von 0,09 auf $0,07 \mathrm{~mm}$ verdunnte Stelle, beide mit deutlichem Markschwund, ein weißes Haar zeigte in $32 \mathrm{~mm}$ Entfernung eine $4 \mathrm{~mm}$ lange, marklose resp. nur stellenweise mit Mark versehene Stelle. Das Haarwachstum betrug also in den 75 Tagen $0,44,0,39$ resp. $0,43 \mathrm{~mm}$ pro Tag und in den Tagen der 56 tägigen Erkrankung 0,1,0,03 resp. 0,08 mm.

Es geht daraus hervor, daß unter Umständen (wie es wohl meist der Fall sein" dürfte) die Mehrzahl der Haare eine Grippeerkrankung überstehen kann und bei mehrmaliger Erkrankung eventuell erst bei der zweiten Schädigung zugrundegeht. Auch diese Tatsache müBte sich später am Haare noch ablesen lassen. Dazu glaube ich folgenden Beleg gefunden zu haben:

A. Th. (Beobachtung 8, J.-Nr. 2737), 13 Jahre alt, dunkelbraun, litt Anfang Oktober acht Tage lang an Grippe, woran sich nach vorübergehender Besserung eine fünftägige Lungenentzündung (mit vier Tage währendem Fieber von $41^{\circ}$ ) anschloß, Patientin kam am 27. Januar wegen seit fünf bis sechs Tagen bestehenden Haarausfalls in Behandlung. Die Differenz zwischen Ende der Erkrankung und Haarausfall betrug also etwa 90 Tage. Mikroskopisch fanden sich mehrere Kolbenhaare mit doppelter Depigmentierung. So war ein markloses Haar vom Wurzelende an zunächst $1 \mathrm{~mm}$ lang depigmentiert, dann $2,4 \mathrm{~mm}$ pigmentiert, dann wieder heller, das Dickenmaß betrug gleichmäßig $0,05 \mathrm{~mm}$, ein anderes, gleichfalls marklos̈es Haar 1,6 mm lang depigmentiert, dann 1,6 mm pigmentiert, dann heller, die Dickenmaße betrugen hier an der Stelle der ersten Aufhellung $0,0625 \mathrm{~mm}$, dann gleichbleibend $0,075 \mathrm{~mm}$. Man dürfte dieses Bild auf den vorübergehenden Rückgang der Erkrankung zurückführen. 
Zusammenfassend kann man sagen, daß die. Grippeerkrankung zu einer Ernährungs- und Wachstumsstörung des Haares führt, die in manchen Fällen offenbar als foudroyante Toxinwirkung - analog der Einwirkung auf den Herzmuskel - aufzufassen ist und zum subakuten oder akuten Absterben der Papille führt und deren Folgen beim überlebenden Haar in meßbarer Länge und Entfernung vom Wurzelende ein untrügliches Zeichen der überstandenen Erkrankung darbieten.

Genauere histologische Untersuchungen der Kopfhaut in dergleichen. Fällen scheinen selten ausgeführt worden zu sein. Wir haben dabei keine wichtigen Befunde erhoben. Es ist bekannt, daß die nach Eintritt des Haartodes sich ausbildenden verämderten Druckverhältnisse im Follikel einen Reiz zum Wachstum des neuen Haares bilden, welches nach zwei bis drei Monaten eine solche Stärke erreicht hat, daß es in dem bereits mechanisch erweiterten Follikel den letzten Halt des Kolbenhaares durch Verdrängung vollends löst. Die mitunter fast momentane Einwirkung der Schädigung führt nach $2^{1} / 2-3$ Monaten infolge einer fast gleichzeitig einsetzenden Lockerung der Haare zu dem mitunter gleichfalls schlagartig einsetzenden Haarverlust.

So werden die Bedingungen verständlich, weshalb meist kurz nach dem Verlust der alten das Erscheinen der neuen Haare einsetzt. Immerhin dauert es, wie bereits Pinkus ${ }^{i}$ ) für die Alopezie nach fieberhaften Erkrankungen hervorhob, bei dem durchschnittlichen Wachstum der Haare von täglich $0,4 \mathrm{~mm}$ ein Jahr, bis eine Länge von $18 \mathrm{~cm}$ erreicht ist, und fünf bis sechs Jahre, bis auch die längsten Haare ersetzt sind. Die Prognose ist aber mit dieser Einschränkung günstig.

Therapeutisch haben wir durch Allgemeinbehandlung mit El a r s o n, lokal infolge einer durch Höhensonne bewirkten entzündlichen Gewebsreaktion die Haarfollikel unter bessere Ernährungsbedingungen zu setzen versucht, was bei gleichzeitiger Anwendung eines Haarwassers zu günstigen Resultaten führte; als solches benutzten wir vorwiegend: $:^{2}$ )
Acid. salic.
Menthol aa 1,0
Sublimat 0,4
Resorcin alb. aa $4,0-5,0$
Anthrasol 10,0
Perkaglyzerin $1-3,0$
Spiritus vini ad 200,0
Spirit. vini $(70 \%)$ ad 200,0 